\title{
International Journal of Applied Mathematics
}

\section{Volume 26 No. 2 2013, 203-220}

ISSN: 1311-1728 (printed version); ISSN: 1314-8060 (on-line version) doi: http://dx.doi.org/10.12732/ijam.v26i2.7

\section{A NUMERICAL METHOD TO COMPUTE THE VOLATILITY OF THE FRACTIONAL BROWNIAN MOTION IMPLIED BY AMERICAN OPTIONS}

\author{
Luca Vincenzo Ballestra ${ }^{1}$, Liliana Cecere $^{2}$ \\ ${ }^{1,2}$ Dipartimento di Economia \\ Seconda Università di Napoli \\ Corso Gran Priorato di Malta, 81043, Capua, ITALY
}

\begin{abstract}
We develop a highly efficient approach to compute the volatility of the Fractional Brownian Motion (FBM) implied by American options. To this aim, the theoretical values of American option prices under the FBM are calculated using a finite difference scheme enhanced by a space-time Richardson extrapolation procedure. Such an approach, which is coupled with a suitable optimization algorithm, turns out to be very accurate and fast. The practical performances of the method proposed are demonstrated by applying it to the time-series of several stocks belonging to the Italian FTSE-All Share.
\end{abstract}

AMS Subject Classification: 65B05, 65N06, 91G60, 91G80

Key Words: fractional Brownian Motion, option pricing, implied volatility, finite difference method, Richardson extrapolation

\section{Introduction}

The Fractional Brownian Motion (FBM), originally introduced by Mandelbrot and Van Ness, is an improvement of the well-known Geometric Brownian Motion $(\mathrm{GBM})$, on which the famous Black-Scholes option pricing model stands [12]. In particular, the GBM has the drawback of not considering the past history of stock returns, that is the yield of a security at any given time is totally

Received: March 28, $2013 \quad$ (c) 2013 Academic Publications

${ }^{\S}$ Correspondence author 
uncorrelated with the returns at the previous times. By contrast, the FBM allows one to model the dependence of a stochastic process on its past history (long-range dependence) and thus it is generally considered more accurate than the GBM in describing asset prices, which normally exhibit a high degree of autocorrelation (see for example [11, 22, 30, 32, 40, 53, 57]).

In this paper we propose an efficient method to estimate the volatility of the FBM implied by American option prices. Note that it is particularly important to take into account American options (rather than European options), as the majority of the options traded on the markets are of this type.

Computing the volatility of the FBM implied by American options is a challenging problem. In fact, American option prices under the FBM are not available in closed form; so, one has to employ an approximate solution, but such an approximate solution must be very accurate and fast, because, in order to obtain a reliable estimation of the implied volatility (for example by least squares fitting), American option prices need to be calculated several times and with enough precision.

To the best of our knowledge, the problem of estimating the volatility of the FBM implied by American option prices has been addressed only in [28]. In particular, in that work, the theoretical values of the option prices are computed using two different approximate solutions, namely the well-known formula by [7] and the formula proposed by [36]. Now, the paper [28] is certainly interesting, as it is (again to the best of our knowledge) the only published work dealing with the estimation of the volatility of the FBM implied by American options. Nevertheless, the estimation approach proposed therein is not particularly accurate. In fact, first of all, the volatilities obtained using the two formulae (that by [7] and that by [36]) are sometimes significantly different one from the other (the relative difference can also be the ten percent, see [28]), which makes it difficult to tell whether and which of the two formulae provides a sharp estimation of the implied volatility. Second, [28] make a rough approximation, as the early exercise premium typical of American options is calculated using the standard formulae of Black and Scholes, which are valid for the GBM but not for the FBM.

In the present paper, an efficient estimation of the volatility of the FBM implied by American options is obtained by employing an ad-hoc numerical discretization/optimization procedure. Precisely, first of all a fast and accurate approximation of American option prices under the FBM is computed by means of a finite difference scheme enhanced by a space/time Richardson extrapolation technique. Then, the problem of minimizing the distance between theoretical and empirical option prices is solved using the Brent's algorithm (see [14]). This 
latter approach turns out to be very appropriate as it does not require the use of derivatives, which would not be available since the American option prices are computed by finite difference approximation.

In summary, the method proposed greatly reduces the complexity of the problem, so that the volatility of the FBM implied by American options can be calculated very accurately and quickly. In fact, as shown by the numerical experiments reported in Section 4, on a normal personal computer the volatility is obtained with at least three correct decimal digits in only a couple of seconds.

We point out that the main purpose of this paper is to develop a highly efficient approach to estimate the volatility implied by American option prices under the FBM. However, as a sub-product of this work, we also develop a numerical method to compute American option prices under the FBM (and thus under the GBM as well) which reveals to be very accurate and fast. This method could also be employed for valuing American options on underlying asset prices described by models other than the FBM, such as, for example, the CEV model [18], or models with jumps [45].

The paper is organized as follows: in Section 2 the basic facts of the FBM are recalled; in particular, our attention is focused onto the partial differential problem that allows us to compute the price of American options; in Section 3 the method to estimate the implied volatility is developed; in Section 4 the numerical results are presented and a numerical comparison between the FBM and the GBM is provided; finally, in Section 5 some conclusions are drawn.

\section{Fractional Brownian Motion}

The FBM is a Gaussian process with zero mean and mutually independent stationary increments. In particular, the structure of increments is modeled by a fractional Wiener process $[21,43,52]$ :

$$
\begin{aligned}
B_{H}(t)=B_{H}(0)+\frac{1}{\Gamma\left(H+\frac{1}{2}\right)}\left[\int _ { - \infty } ^ { 0 } \left((t-s)^{H-\frac{1}{2}}\right.\right. & \left.-(-s)^{H-\frac{1}{2}}\right) d W(s) \\
& \left.+\int_{0}^{t}(t-s)^{H-\frac{1}{2}} d W(s)\right],
\end{aligned}
$$

where $W$ is a standard Wiener process [58] and $\Gamma$ is the Gamma function [1]. In (1) $H$ is the so-called Hurst exponent, which takes values in $(0,1)$.

The FBM is often used to describe the prices of stocks traded in the financial markets (see for example [11, 30, 32, 40, 57]). In particular, it is assumed that, 
under the dividend adjusted risk-neutral measure (see [58]), the price of a stock $S(t)$ satisfies the stochastic differential equation:

$$
d S(t)=(r-q) S(t) d t+\sigma S(t) \diamond d B_{H}(t),
$$

where $r$ is the interest rate, and $q$ and $\sigma$ are the (continuous) dividend yield and the volatility of the stock respectively. In $(2)$ the symbol $\diamond$ denotes the so-called Wick product (see [31]).

The Hurst exponent is a parameter that characterizes the FBM: if $H=\frac{1}{2}$ the process (1) degenerates to the standard Wiener process (so that $S$ reduces to the well-known GBM); if $H>\frac{1}{2}$ the increments of the process (1) have positive autocorrelation (so that $S$ has a persistent trend); if $H<\frac{1}{2}$ the increments of the process (1) have negative autocorrelation (so that $S$ has an anti-persistent trend).

\subsection{American Option Pricing under the FBM}

The great majority of the traded options are of American style. This is due to the fact that American options can be exercised at any time on or before the expiration date, and thus are considered very appealing by investors.

The price of an American option under the FBM satisfies a partial differential problem which is described in the following. For the sake of brevity, we only consider the case of American Call options. Nevertheless, an analogous partial differential problem can be written for American Put options as well.

Let $C(S, t)$ denote the price at time $t$ of an American Call option on an underlying price $S$, with maturity $T$ and strike price $K$. It can be shown (see, for example, [6]) that $C(S, t)$ satisfies the following partial differential problem:

$$
\begin{gathered}
\frac{\partial C(S, t)}{\partial t}+\frac{1}{2} H^{2} \sigma^{2} S^{2} \frac{\partial^{2} C(S, t)}{\partial S^{2}}+(r-q) S \frac{\partial C(S, t)}{\partial S}-r C(S, t), \\
C(S, T) \geq \phi(S), \\
\left(\frac{\partial C(S, t)}{\partial t}+\frac{1}{2} H^{2} \sigma^{2} S^{2} \frac{\partial^{2} C(S, t)}{\partial S^{2}}+(r-q) S \frac{\partial C(S, t)}{\partial S}-r C(S, t)\right) \\
\times(C(S, t)-\phi(S))=0,
\end{gathered}
$$

with final condition:

$$
C(S, T)=\phi(S)
$$


and boundary conditions:

$$
C(0, t)=0, \quad C(S, t) \sim S-K \quad \text { as } \quad S \rightarrow+\infty .
$$

In (4), (5) and (6) the function $\phi$ is the so-called option's payoff:

$$
\phi(S)=\max [S-K ; 0] .
$$

Problem (3)-(7) is rather complex from the analytical standpoint. In particular, no exact closed-form solution is available and numerical approximation is required.

\section{The Estimation Method}

The calculation of the implied volatility is done in three steps. First of all, the Hurst exponent, which is needed in problem (3)-(7), is estimated; second, an accurate and fast approximation of American option prices under the FBM is computed; third, the implied volatility is obtained as the value that minimizes the distance between the theoretical and the empirical American option prices.

\subsection{Hurst Exponent}

To estimate the Hurst parameter $H$ of the FBB several different approaches have been proposed. For example, [4, 17, 26, 32, 33, 35, 40, 42, 44, 47, 49, 50, 59] use the so-called Rescaled Range analysis (R/S), [2, 3, 9, 38] apply wavelet transform techniques, $[10,46,55,56]$ employ maximum likelihood estimation, and $[8,19,20,37]$ have developed a special procedure called multi-fractal analysis, according to which the parameter $H$ is related to the moments of the probability distribution of the increments of (1).

In the present paper we decide to employ the $\mathrm{R} / \mathrm{S}$ analysis, for mainly two reasons: first of all, among all the various approaches, the $\mathrm{R} / \mathrm{S}$ analysis is the most common one when dealing with time series of asset returns (see for example, $[4,17,26,32,40,44,47,50])$; second, such a technique, besides being simple to implement and computationally fast, provides a very accurate and robust estimation of the Hurst exponent. In particular, as the number of observations increases, the $R / S$ estimator converges almost surely (i.e. with probability one) to the true value of $H$ (see [39]).

Nevertheless, we make observe that the method used to compute the Hurst parameter is not the main focus of our paper, as we are primarily concerned 
with estimating the implied volatility of the FBM. Then, methods other than the $\mathrm{R} / \mathrm{S}$ analysis, such as some of those mentioned above, could be employed as well.

Let us briefly recall how the R/S analysis works (for details see [39]). Let $S_{0}, S_{1}, \ldots, S_{N_{x}}$ denote the prices of a stock observed at times $t_{0}, t_{1}, \ldots, t_{N_{x}}$, respectively. Let us define the logarithmic returns:

$$
x_{i}=\ln \frac{S_{i}}{S_{i-1}}, \quad i=1,2, \ldots, N_{x} .
$$

The $R / S$ statistic is defined as follows:

$$
R / S=\frac{1}{2}\left[\max _{k \in 1,2, \ldots, N_{x}} \sum_{i=1}^{k}\left(x_{i}-\bar{x}\right)-\min _{k \in 1,2, \ldots, N_{x}} \sum_{i=1}^{k}\left(x_{i}-\bar{x}\right)\right],
$$

where

$$
\bar{x}=\frac{1}{N_{x}} \sum_{i=1}^{N_{x}} x_{i} .
$$

The estimated value of $H$, which we denote $H^{*}$, is obtained as the slope of the linear regression of $\ln [R / S]$ on $\ln \left(N_{x}\right)$.

\subsection{Numerical Approximation of American Option Prices}

As already observed, problem (3)-(7), which yields the prices of American options under the FBM, requires numerical approximation. In this paper, we consider a time discretization of it that amounts to computing American options prices by Richardson extrapolation of the prices of Bermudan options; let us recall that a Bermudan option is an option that can be exercised only at a discrete set of dates. The advantages of such an approach have been assessed, for instance, in $[5,6,15,16,25,29,41]$; in particular, the Richardson extrapolation is computationally faster than methods based on complementarity formulations, such as the linear complementarity method $[13,34]$ or the penalty method $[23,24]$, which require one to perform a fixed-point iteration at every time step.

In the interval $[0, T]$ let us consider $N_{t}+1$ equally spaced time levels $t_{0}, t_{1}, \ldots, t_{N_{t}}$ such that $t_{k}=k \Delta t, k=0,1, \ldots, N_{t}$, where $\Delta t=\frac{T}{N_{t}}$. Let $C_{\Delta t}(S, t)$ denote the price of a Bermudan option with maturity $T$ and exercise dates $t_{0}, t_{1}, \ldots, t_{N_{t}}$. The Bermudan option price $C_{\Delta t}(S, t)$ is obtained using the following recursion procedure. First of all set $k=N_{t}$, and define:

$$
\Psi\left(S, t_{k}\right)=\phi(S) .
$$


Then, for $t \in\left[t_{k-1}, t_{k}\right)$, solve the partial differential problem:

$$
\begin{gathered}
\frac{\partial C_{\Delta t}(S, t)}{\partial t}+\frac{1}{2} H^{2} \sigma^{2} S^{2} \frac{\partial^{2} C_{\Delta t}(S, t)}{\partial S^{2}}+(r-q) S \frac{\partial C_{\Delta t}(S, t)}{\partial S}-r C_{\Delta t}(S, t)=0, \\
C_{\Delta t}(0, t)=0, \quad C_{\Delta t}(S, t) \sim S-K \quad \text { as } \quad S \rightarrow+\infty, \\
C_{\Delta t}\left(S, t_{k}\right)=\Psi\left(S, t_{k}\right) .
\end{gathered}
$$

Then set:

$$
\Psi\left(S, t_{k-1}\right)=\max \left[C_{\Delta t}\left(S, t_{k-1}\right), \phi(S)\right] .
$$

Finally, update the counter:

$$
k:=k-1,
$$

and repeat the cycle (13)-(17) until $k=0$.

Problem (13)-(16) corresponds to performing a time discretization of the possibility of early exercise by imposing the constraint (16) only at the dates $t_{1}, t_{2}, \ldots, t_{N_{t}}$. The Bermudan option price $C_{\Delta t}$ tends to become a fair approximation of the American option price $C$ as the number of exercise dates $N_{t}$ increases or, alternatively, as $\Delta t \rightarrow 0$. In particular, as shown in [29], $C_{\Delta t}$ tends to $C$ like $O(\Delta t)$ as $\Delta t \rightarrow 0$.

This convergence rate is not very high and thus is enhanced by Richardson extrapolation. In particular, in the present work we employ both the linear Richardson extrapolation:

$$
C(S, t) \cong 2 C_{2 \Delta t}(S, t)-C_{\Delta t}(S, t),
$$

and the quadratic Richardson extrapolation:

$$
C(S, t) \cong \frac{8 C_{4 \Delta t}(S, t)-6 C_{2 \Delta t}(S, t)+C_{\Delta t}(S, t)}{3},
$$

which are second-order accurate and third-order accurate as $\Delta t \rightarrow 0$, respectively (see $[27]$ ).

Finally, it remains to solve the partial differential problem (13)-(15). To this aim, we approximate the time derivative in (13) through a single step of the implicit Euler finite difference scheme (whose first-order convergence is enhanced thanks to the extrapolations (18) and (19)). Precisely, let $C_{\Delta t}^{k}(S)$ and $\Psi_{\Delta t}^{k}(S)$ denote approximate values of $C_{\Delta t}\left(S, t_{k}\right)$ and $\Psi_{\Delta t}\left(S, t_{k}\right)$, respectively. Problem (13)-(15) is discretized in time as follows:

$$
\begin{aligned}
\frac{\Psi_{\Delta t}^{k}(S)-C_{\Delta t}^{k-1}(S)}{\Delta t}+\frac{1}{2} H^{2} \sigma^{2} S^{2} \frac{\partial^{2} C_{\Delta t}^{k-1}(S)}{\partial S^{2}}+(r-q) & \frac{C_{\Delta t}^{k-1}(S)}{\partial S} \\
& -r C_{\Delta t}^{k-1}(S)=0
\end{aligned}
$$




$$
C_{\Delta t}^{k-1}(0)=0, \quad C_{\Delta t}^{k-1}(S) \sim S-K \quad \text { as } \quad S \rightarrow+\infty .
$$

Finally, we discretize problem (20)-(21) in the $S$ variable. This is done using a finite difference approach which, once again, employs the Richardson extrapolation procedure.

First of all, the infinite spatial domain $[0,+\infty)$ is replaced with a bounded one $\left[0, S_{\max }\right]$, where $S_{\max }$ is chosen large enough so that the truncation error is negligible (as is customary we set $S_{\max }=2 K$ ).

Then let us consider $N_{S}$ equally spaced points $S_{j}=(j-1) h, j=1,2, \ldots, N_{S}$, where $\Delta S=\frac{S_{\max }}{N_{S}-1}$, and let $C_{\Delta t, \Delta S}^{k-1, j}$ denote an approximate value of $C_{\Delta t}^{k-1}\left(S_{j}\right)$, $j=1,2, \ldots, N_{S}$. Equation (20) is discretized in space using the (centered) threepoint finite difference scheme [51]:

$$
\begin{aligned}
\frac{\Psi_{\Delta t}^{k, j}-C_{\Delta t, \Delta S}^{k-1, j}}{\Delta t}+ & (r-q) S_{j} \frac{C_{\Delta t, \Delta S}^{k-1, j+1}-C_{\Delta t, \Delta S}^{k-1, j-1}}{\Delta S} \\
& +\frac{1}{2} H^{2} \sigma^{2} S_{j}^{2} \frac{C_{\Delta t, \Delta S}^{k-1, j+1}-2 C_{\Delta t, \Delta S}^{k-1, j}+C_{\Delta t, \Delta S}^{k-1, j-1}}{\Delta S^{2}} \\
& -r C_{\Delta t, \Delta S}^{k-1, j}=0, \quad j=2,3, \ldots, N_{S}-1 .
\end{aligned}
$$

The boundary conditions (21) are simply imposed as follows:

$$
C_{\Delta t, \Delta S}^{k-1,1}=0, \quad C_{\Delta t, \Delta S}^{k-1, N_{S}}=S_{\max }-K .
$$

Relations (22)-(23) constitute a tridiagonal system of linear equations in the unknowns $C_{\Delta t, \Delta S}^{k-1,1}, C_{\Delta t, \Delta S}^{k-1,2}, \ldots, C_{\Delta t, \Delta S}^{k-1, N_{S}}$, which is solved very quickly by means of the well known Thomas algorithm (see [54]). According to the recursion (13)-(17), the numerical scheme (22)-(23) is repeated at every time-step $t_{k}$, $k=N_{t}, N_{t-1}, \ldots, 1$, so that we end up with the values $C_{\Delta t, \Delta S}^{k, 1}, C_{\Delta t, \Delta S}^{k, 2}, \ldots, C_{\Delta t, \Delta S}^{k, N_{S}}$, for every $k \in\left\{0,1, \ldots, N_{t}\right\}$.

The approximations $C_{\Delta t, \Delta S}^{k, 1}, C_{\Delta t, \Delta S}^{k, 2}, \ldots, C_{\Delta t, \Delta S}^{k, N_{S}}$ become more and more accurate as the number of discretization points $N_{S}$ increases, or, equivalently, as $\Delta S$ tends to zero. Precisely, $C_{\Delta t, \Delta S}^{k, 1}, C_{\Delta t, \Delta S}^{k, 2}, \ldots, C_{\Delta t, \Delta S}^{k, N_{S}}$ converge to

$$
C_{\Delta t}^{k}\left(S_{1}\right), C_{\Delta t}^{k}\left(S_{2}\right), \ldots, C_{\Delta t}^{k}\left(S_{N_{S}}\right)
$$

like $O\left(\Delta S^{2}\right)$ as $\Delta S \rightarrow 0$ (see [51]). Therefore, it is possible to remove the $O\left(\Delta S^{2}\right)$ error term using the (quadratic) Richardson extrapolation procedure $[27]:$

$$
C_{\Delta t, \Delta S, \text { extr }}^{k, j}=\frac{4 C_{\Delta t, \Delta S / 2}^{k, 2 j-1}-C_{\Delta t, \Delta S}^{k, j}}{3}, \quad j=1,2, \ldots, N_{S},
$$


where $C_{\Delta t, \Delta S / 2}^{k, \ell}, \ell=1,2, \ldots, 2 N_{S}-1$, denote the approximate option prices obtained using a finite difference mesh of $2 N_{S}-1$ nodes (i.e. with grid spacing $\Delta S / 2$ ).

The values $C_{\Delta t, \Delta S \text {, extr }}^{k, j}, j=1,2, \ldots, N_{S}$, are extremely accurate approximations of the true American option prices. In particular, the spatial discretization error (in the $S$ variable) tends to zero faster than $\Delta S^{2}$ as $\Delta S$ tends to zero.

Finally, we note that the numerical method described above provides an approximation of the American option price only at the nodes $S_{1}, S_{2}, \ldots, S_{N_{S}}$. Then, for values of $S$ that do not coincide with the mesh nodes, the American option price is obtained by cubic extrapolation of the option prices at the four nodes closest to $S$ (which is extremely quick and does not compromise the $o\left(\Delta S^{2}\right)$ accuracy of the spatial extrapolation scheme described above).

\subsection{Implied Volatility Estimation}

Let $V_{1}, V_{2}, \ldots, V_{N_{o p}}$ denote realized prices of American options. In particular, let $V_{1}, V_{2}, \ldots, V_{N_{o p}}$ be the prices (observed at different times) of a set of options written on the same underlying stock but having different strikes and maturities. Moreover, let $V_{1}^{A P}(\sigma), V_{2}^{A P}(\sigma), \ldots, V_{N_{o p}}^{A P}(\sigma)$ denote the corresponding values of the theoretical prices under the FBM (computed according to the numerical method described in Section 3.2). Note that as explicitly stated, the quantities $V_{1}^{A P}, V_{2}^{A P}, \ldots, V_{N_{o p}}^{A P}$ depend on the volatility parameter $\sigma$.

Let us define the objective function:

$$
G(\sigma)=\sum_{i=1}^{N_{o p}}\left(V_{i}-V_{i}^{A P}(\sigma)\right)^{2},
$$

which measures the distance between the theoretical and the empirical option prices. We want to find the value of $\sigma$ that minimizes $G$, i.e. the implied volatility $\sigma_{F B M}^{*}$ is obtained such that:

$$
\sigma_{F B M}^{*}=\arg \min _{\sigma>0} G(\sigma) .
$$

Problem (26) is solved using the numerical optimization algorithm developed by Brent [14], which is based on a quadratic approximation of the objective function $G$. For a detailed description of this method the reader is reminded to [48]; here, we simply observe that the Brent's algorithm does not require knowledge of the derivative of $G$ with respect to $\sigma$ (which is not available since the theoretical option prices are obtained by finite difference approximation), and thus turns out to be very suitable to solve problem (26). 
Remark. We do not perform a theoretical investigation of the convergence of the numerical estimation method developed in this section. In fact, such an analysis would be an extremely difficult task, due to the many different techniques employed (the Bermudan approximation, the finite difference scheme, the space/time Richardson extrapolation, the Brent's algorithm), and goes beyond the scope of this paper. Therefore, as is customary when dealing with implied volatility estimation, the computational properties of the algorithm described above are directly tested by numerical approximation. This is done in the next section.

\section{Numerical Results}

The numerical simulations are performed on a personal computer with an Athlon II X2 Dual Core Processor 1.3 GHZ 3.00 GB Ram and the software codes are written in Matlab 7.0.

We compute the volatility of the FBM for 12 equities of the FTSE-All Shares Italian market, namely: Autogrill (AGL), Atlantia (ATL), Eni (ENI), Fiat (F), Finmeccanica (FNC), Generali (G), Intesa Sanpaolo (ISP), Lottomatica (LTO), Luxottica (LUX), Saipem (SPM), Stmicroelectronics (STM), Unicredit (UCG). For each one of these equities we calculate the Hurst exponent based on approximately 1200 daily prices observed from 2nd January 2006 to 1st December 2010 (i.e. $N_{x} \cong 1200$ ). This set of data is very large. Therefore, upon the almost sure convergence of the $R / S$ estimator (see Section 3 ) the value $H^{*}$ is deemed statistically accurate. Moreover, for each one of the above equities we calculate the implied volatility using both Call and Put American options with various different strikes and maturities equal to 3, 6 and 12 months. In particular, our database of options covers the period of time from 4th January 2010 to 1 st December 2010, so for each one of the 12 equities considered we have a number of option prices that ranges approximately from 200 to 2000 (i.e. $200<N_{o p}<2000$ ).

Following a very common approach, the (continuous) dividend yield $q$ (see equation (2)) is determined by imposing the equivalence between the discounted actual value of the continuous dividend flow and the discounted actual value of the discrete dividends that have been paid in the time period from 4th January 2010 to 1 st December 2010. As far as the interest rate $r$ is concerned, we use the 12 month Euribor rate at 1st December 2010, as many of the options considered expire between 1st December 2010 and 1st December 2011.

However, we have performed several numerical experiments (some of which 
are not reported in the paper) in which we have used different (and reasonable) values of $q$ and $r$, and we have found that the effect of these two parameters on the implied volatility is negligible.

The estimates $H^{*}$ and $\sigma^{*}$ are shown in Table 1, in which, for the sake of completeness, we also report the value of the objective function at $\sigma^{*}$. In particular, the parameters in Table 1 are obtained using the finite difference method with 41 spatial nodes $\left(N_{S}=41\right)$ and with cubic Richardson extrapolation in time (relation (19)), which, as shown in the following, allows us to compute the implied volatility with at least 3 correct decimal digits.

\begin{tabular}{|l|c|c|c|c|}
\hline Equity & $q$ & $H^{*}$ & $\sigma^{*}$ & $G\left(\sigma^{*}\right)$ \\
\hline AGL & 0.019 & 0.5556 & 0.2863 & 0.1044 \\
\hline ATL & 0.098 & 0.5224 & 0.2620 & 4.8667 \\
\hline ENI & 0.096 & 0.5034 & 0.2400 & 13.1277 \\
\hline F & 0.029 & 0.5175 & 0.3696 & 15.6705 \\
\hline FNC & 0.038 & 0.5530 & 0.2495 & 20.4664 \\
\hline G & 0.024 & 0.5865 & 0.2675 & 7.1418 \\
\hline ISP & 0.035 & 0.6448 & 0.3348 & 3.6539 \\
\hline LTO & 0.048 & 0.5877 & 0.2346 & 42.8268 \\
\hline LUX & 0.023 & 0.5073 & 0.2296 & 0.0661 \\
\hline SPM & 0.030 & 0.5326 & 0.2851 & 17.6876 \\
\hline STM & 0.029 & 0.5658 & 0.3075 & 0.5020 \\
\hline UCG & 0.017 & 0.6085 & 0.3492 & 0.4841 \\
\hline
\end{tabular}

Table 1: Estimation of the parameters of the FBM, $N_{S}=41$, quadratic extrapolation in time

Looking at the Table 1, we may note that the values of $H^{*}$ are between 0.5034 and 0.6448 , so all the equities considered have a persistent trend. Furthermore, the volatility $\sigma^{*}$ takes values between 0.2296 and 0.3696 , which are typical values of volatility.

We have also analyzed the dependence of $\sigma^{*}$ on the mesh size parameter $N_{S}$ and on the order of the Richardson extrapolation in time (two or three, corresponding to relations (18) or (19) respectively). In particular, in Table 2 and Table 3 we show the values of $\sigma^{*}$ and the computer times obtained by choosing different values of $N_{S}$ and by using both the linear and the quadratic extrapolation in time (for the sake of brevity, we only report the results concerning ATL and UCG, as the results experienced for all the remaining equities are substantially identical).

Looking at Table 2 and Table 3, we may see that, by choosing $N_{S}=41$ and by using the linear Richardson extrapolation, the implied volatility is obtained 
with at least three correct digits in a time smaller than two seconds. Therefore, the method proposed is accurate and fast. This conclusion is also strengthened by the fact that we are using a significantly large database of option prices $\left(200<N_{o p}<2000\right)$, and thus we have to solve the partial differential problem (13)-(15) a large number of times.

\begin{tabular}{|l|c|c|c|c|}
\hline & \multicolumn{2}{|c|}{ Linear extrapolation in time } & \multicolumn{2}{c|}{ Quadratic extrapolation in time } \\
\hline$N_{S}$ & $\sigma^{*}$ & CPUTime & $\sigma^{*}$ & CPUTime \\
\hline 21 & 0.2610 & $1.48(s)$ & 0.2610 & $2.46(s)$ \\
\hline 41 & 0.2620 & $1.82(s)$ & 0.2620 & $3.61(s)$ \\
\hline 81 & 0.2621 & $2.90(s)$ & 0.2621 & $5.99(s)$ \\
\hline
\end{tabular}

Table 2: Computation of the implied volatility, ATL

\begin{tabular}{|l|c|c|c|c|}
\hline & \multicolumn{2}{|c|}{ Linear extrapolation in time } & \multicolumn{2}{c|}{ Quadratic extrapolation in time } \\
\hline$N_{S}$ & $\sigma^{*}$ & CPUTime & $\sigma^{*}$ & CPUTime \\
\hline 21 & 0.3490 & $1.30(s)$ & 0.3490 & $2.44(s)$ \\
\hline 41 & 0.3492 & $1.79(s)$ & 0.3492 & $3.62(s)$ \\
\hline 81 & 0.3492 & $2.78(s)$ & 0.3492 & $5.11(s)$ \\
\hline
\end{tabular}

Table 3: Computation of the implied volatility, UCG

\section{Comparison with the Black-Scholes model:}

Finally, let us briefly compare the FBM with the GBM, on which is based the famous Black and Scholes model. To this aim, the volatility of the GBM implied by American option prices is estimated using the method developed in Section 3.2 and 3.3, in which we simply set $H=\frac{1}{2}$ (as already recalled, for $H=\frac{1}{2}$ the FBM degenerates to the GBM). In the following, in order to avoid confusion, the volatility of the FBM will be denoted by $\sigma_{F B M}^{*}$, whereas the volatility of the GBM will be denoted by $\sigma_{G B M}^{*}$. As done in [28], let us consider the following indicators:

$$
\epsilon_{\sigma}=\frac{\left|\sigma_{F B M}^{*}-\sigma_{G B M}^{*}\right|}{\sigma_{F B M}^{*}}, \quad \epsilon_{G}=\frac{\left|G\left(\sigma_{F B M}^{*}\right)-G\left(\sigma_{G B M}^{*}\right)\right|}{G\left(\sigma_{F B M}^{*}\right)},
$$

which measure the deviations in the values of volatility and objective function between the FBM and the GBM.

The values of $\epsilon_{\sigma}$ and $\epsilon_{G}$ obtained for the 12 equities considered so far are shown in Table 4. We may note that both $\epsilon_{\sigma}$ and $\epsilon_{G}$ are very small. In 
particular, the (relative) difference between the volatility of the FBM and the volatility of the GBM is always smaller than $4.63 \times 10^{-2}$; in addition, we also observe that the objective function does not change significantly when passing from the GBM to the FBM (the maximum value of $\epsilon_{G}$ is $8.71 \times 10^{-2}$ ). Therefore, we conclude that the FBM provides only a small improvement over the GBM as far as the pricing of American options is concerned.

\begin{tabular}{|l|c|c|}
\hline Equity & $\epsilon_{\sigma}$ & $\epsilon_{G}$ \\
\hline AGL & $2.79 \times 10^{-2}$ & $1.83 \times 10^{-2}$ \\
\hline ATL & $5.77 \times 10^{-3}$ & $2.20 \times 10^{-2}$ \\
\hline ENI & $7.89 \times 10^{-4}$ & $1.88 \times 10^{-3}$ \\
\hline F & $7.46 \times 10^{-3}$ & $2.48 \times 10^{-3}$ \\
\hline FNC & $1.76 \times 10^{-2}$ & $1.70 \times 10^{-3}$ \\
\hline G & $1.23 \times 10^{-3}$ & $8.71 \times 10^{-2}$ \\
\hline ISP & $4.63 \times 10^{-2}$ & $7.15 \times 10^{-2}$ \\
\hline LTO & $2.58 \times 10^{-2}$ & $1.02 \times 10^{-3}$ \\
\hline LUX & $9.24 \times 10^{-3}$ & $5.48 \times 10^{-3}$ \\
\hline SPM & $1.23 \times 10^{-2}$ & $4.65 \times 10^{-2}$ \\
\hline STM & $1.62 \times 10^{-2}$ & $5.80 \times 10^{-3}$ \\
\hline UCG & $3.69 \times 10^{-2}$ & $2.56 \times 10^{-2}$ \\
\hline
\end{tabular}

Table 4: Comparison between the FBM and the GBM

\section{Conclusions}

We propose a new and highly efficient approach to compute the volatility of the FBM implied by American option prices. To this aim, the theoretical values of American option prices under the FBM are obtained using a finite difference scheme enhanced by a suitable space-time Richardson extrapolation procedure. Such an approach, which is used in conjunction with the Brent's optimization algorithm, allows us to considerably reduce the complexity of the overall estimation problem. Thus, the volatility of the FBM is evaluated very accurately and quickly (on a normal personal computer the implied volatility is obtained with at least three correct digits in only a couple of seconds). The computational performances of the method proposed are demonstrated by applying it to the time-series of several stocks belonging to the Italian FTSE-All Share. From the modeling standpoint, these simulations highlight that the FBM provides only a small improvement over the more conventional GBM. 


\section{References}

[1] M. Abramowitz and I.A. Stegun, Handbook of Mathematical Functions with Formulas, Graphs, and Mathematical Tables, Dover Publications, New York (1972).

[2] P. Abry and D. Veitch, Wavelet analysis of long range dependent traffic, IEEE Transactions on Information Theory, 44 (1998), 2-15.

[3] M. Akay and R. Fischer, Fractal analysis of HRV signals: A comparative study, Methods of Information in Medicine, 36 (1997), 271-273.

[4] K. Aydogan and G.G. Booth, Are there long cycles in common stock returns? Southern Economic Journal, 55 (1988), 141-149.

[5] L.V. Ballestra and G. Pacelli, Pricing European and American options with two stochastic factors: A highly efficient radial basis function approach, Journal of Economic Dynamics and Control, 37 (2013), 1142-1167.

[6] L.V. Ballestra and C. Sgarra, The evaluation of American options in a stochastic volatility model with jumps: An efficient finite element approach, Computers and Mathematics with Applications, 60 (2010), 15711590 .

[7] G. Barone Adesi and R.E. Whaley, Efficient analytic approximation of American option values, The Journal of Finance, 42 (1997), 301-320.

[8] J. Barunik and L. Kristoufek, On Hurst exponent estimation under heavytailed distributions, Physica A, 389 (2010), 3844-3855.

[9] E. Bayraktar, H.V. Poor and K.R. Sircar, Estimating the fractal dimension of the S\&P 500 Index using wavelet analysis, International Journal of Theoretical and Applied Finance, 7 (2004), 613-643.

[10] J. Beran, A goodness-of-fit test for time series with long range dependence, Journal of the Royal Statistical Society: Series B, 54 (1992), 749-760.

[11] E. Berg and J. Lyagen, Short and long-run dependence in Swedish stock returns, Applied Financial Economics, 8 (1998), 435-443.

[12] F. Black and M. Scholes, The pricing of options and corporate liabilities, Journal of Political Economy, 81 (1973), 637-659. 
[13] M.I. Brennan and E.S. Schwartz, The evaluation of American Put options, Journal of Finance, 32 (1977), 449-462.

[14] R.P. Brent, Algorithms for Minimization without Derivatives, PrenticeHall, New York (1973).

[15] C.C. Chang, S.L. Chung and R.C. Stapleton, Richardson extrapolation techniques for pricing American-style options, Journal of Futures Markets, 27 (2007), 791-817.

[16] C.C. Chang, J.B. Lin, W.C. Tsai and Y.H. Wang, Using Richardson extrapolation techniques to price American options with alternative stochastic processes, Review of Quantitative Finance and Accounting, 39 (2012), 383-406.

[17] Y.W. Cheung, A search for long memory in international stock market returns, Journal of International Money and Finance, 14 (1995), 597-615.

[18] J. C. Cox, S. A. Ross, The valuation of options for alternative stochastic processes, Journal of Financial Economics, 3 (1976), 145-166.

[19] T. Di Matteo, Multi-scaling in finance, Quantitative Finance, 7 (2007), 21-36.

[20] T. Di Matteo, T. Aste and M.M. Dacorogna, Scaling behaviors in differently developed markets, Physica A, 324 (2003), 183-188.

[21] T.E. Duncan, Some aspects of fractional Brownian motion, Nonlinear Analysis: Real World Applications, 47 (2001), 4775-4782.

[22] N. T. Dung, Semimartingale approximation of fractional Brownian motion and its applications, Computers and Mathematics with Applications, 61 (2011), 1844-1854.

[23] P.A. Forsyth and K.R. Vetzal, Quadratic convergence for valuing American options using a penalty method, SIAM Journal on Scientific Computing, 23 (2002), 2095-2122.

[24] P.A. Forsyth, K.R. Vetzal and R. Zvan, A penalty method for American options with stochastic volatility, Journal of Computational and Applied Mathematics, 91 (1998), 199-218.

[25] R. Geske and H.E. Johnson, The American Put option valued analytically, Journal of Finance, 39 (1984), 1511-1524. 
[26] M.T. Greene and B.D. Fielitz, Long term dependence in common stock returns, Journal of Financial Economics, 4 (1977), 339-349.

[27] H. Hairer, S. Norsett and G. Wanner, Solving Ordinary Differential Equations I: Nonstiff Problems, Springer, Berlin (1993).

[28] S.W. Heo, Y. Sarol and J.G. Kang, Valuation of American equity options with quadratic approximation models adopting fractional Brownian motion, Global Business and Finance Review, 15 (2010), 127-140.

[29] S. Howinson, A matched asymptotic expansions approach to continuity corrections for discretely sampled options, part 2: Bermudan options, Applied Mathematical Finance, 14 (2007), 91-104.

[30] D.A. Hsieth, Chaos and non-linear dynamics: applications to financial markets, Journal of Finance, 46 (1991), 1839-1877.

[31] Y. Hu and B. Oksendal, Fractional white noise calculus and applications to finance, Infinite Dimensional Analysis, Quantum Probability and Related Topics, 6 (2003), 1-32.

[32] B. Huang and C. Yang, The fractal structure in multinational stock returns. Applied Economics, 2 (1995), 67-71.

[33] H.E. Hurst, Long term storage capacity of reservoirs, Transactions of the American Society of Civil Engineers, 116 (1951), 770-779.

[34] S. Ikonen and J. Toivanen, Efficient numerical methods for pricing American Put options, Numerical Methods for Partial Differential Equations, 24 (2008), 104-126.

[35] J.L. Jin, J. Cheng and Y.M. Wei, Forecasting flood disasters using an accelerated genetic algorithm: examples of two case studies for China, Natural Hazards, 44 (2008), 85-92.

[36] N. Ju and R. Zong, An approximate formula for pricing American options, Journal of Derivatives, 7 (1999), 31-40.

[37] J.W. Kantelhardt, S.A. Zschiegner, A. Bunde, S. Havlin, E. KoscielnyBunde, and H.E. Stanley, Multifractal detrended fluctuation analysis of nonstationary time series, Physica A, 316 (2002), 87-114. 
[38] L.M. Kaplan and C.C. Jay Kuo, Fractal estimation from noisy data via discrete fractional Gaussian noise (DFGN) and the Haar basis, IEEE Transactions on Signal Processing, 41 (1993), 3554-3562.

[39] W. Li, C. Yu, A. Carriquiry and W. Kliemann, The asymptotic behaviour of the $R / S$ statistics for fractional Brownian motion, Statistics and Probability Letters, 81 (2011), 83-91.

[40] A.W. Lo, Long-term memory in stock market prices, Econometrica, 59 (1991), 1279-1313.

[41] R. Lord, F. Fang, F. Bervoets and C.W. Oosterlee, A fast and accurate FFT-based method for pricing early-exercise options under Levy processes, SIAM Journal on Scientific Computing, 30 (2008), 1678-1705.

[42] B. Mandelbrot and M.S. Taqqu, Robust $R / S$ analysis of long-run serial correlation, Bulletin of the International Statistical Institute, 48 (1979), 69-104.

[43] B. Mandelbrot and J. Van Ness, Fractional Brownian motion, fractional noises and applications, SIAM Review, 10 (1968), 422-437.

[44] M.D. McKenzie, Non-periodic Australian stock market cycles: Evidence from rescaled range analysis, Economic Record, 77 (2001), 393-406.

[45] R.C. Merton, Option pricing when underlying stock returns are discontinuous, Journal of Financial Economics, 3 (1976), 125-144.

[46] B.M. Ninness, Maximum likelihood estimation of the parameters of fractional Brownian motions, In Proc. 34th IEEE Conference on Decision and Control, New Orleans, IEEE, New York (1995), 4018-4023.

[47] E.E. Peters, Chaos and Order in the Capital Markets, John Wiley and Sons, New York (1991).

[48] W.H. Press, B.P. Flannery, S.A. Teukolsky and W.T. Vetterling, Numerical Recipes in Fortran: The Art of Scientific Computing, Cambridge University Press, New York (1992).

[49] G. Rangarajan and D.A. Sant, Fractal dimensional analysis of Indian climatic dynamics, Chaos, Solitons and Fractals, 19 (2004), 285-291. 
[50] S. Sadique and P. Silvapulle, Long-term memory in stock market returns: international evidence, International Journal of Finance and Economics, 6 (2001), 59-67.

[51] D. Tavella and C. Randall, Pricing Financial Instruments: The Finite Difference Approach, Wiley, New York (2000).

[52] T. H. Thao, A note on fractional Brownian motion, Vietnam Journal of Mathematics, 31 (2003), 255-260.

[53] T. H. Thao, An approximate approach to fractional analysis for finance, Nonlinear Analysis: Real World Applications, 7 (2006), 124-132.

[54] L.H. Thomas, Elliptic problems in linear difference equations over a network, Technical Report, Watson Scientific Computing Laboratory, Columbia University, New York (1949).

[55] A. Vidàcs and J.T. Virtamo, Maximum likelihood estimation of the parameters of fractional Brownian traffic with geometrical sampling, In Proc. IFIP TC6 5th Int. Conference on Broadband Communications, Hong Kong, Kluwer, Norwell (1999), 51-62.

[56] A. Vidàcs and J.T. Virtamo, Parameter estimation of geometrically sampled fractional Brownian traffic, In Proc. IEEE INFOCOM 2000, Tel-Aviv, IEEE, New York (2000), 26-30.

[57] X.T. Wang, W.Y. Qiu and F.Y. Ren, Option pricing of fractional version of the Black-Scholes model with Hurst exponent $H$ being in $(1 / 3,1 / 2)$, Chaos, Solitions and Fractals, 12 (2001), 599-608.

[58] P. Wilmott, S. Howison and J. Dewynne, The Mathematics of Financial Derivatives, Cambridge University Press, New York (1995).

[59] X.A. Yin, X.H. Yang and Z.F. Yang, Using the $R / S$ method to determine the periodicity of time series, Chaos, Solitions and Fractals, 39 (2009), 731-745. 